Journal of Biomedical and Pharmaceutical Research

Available Online at www.jbpr.in

CODEN: - JBPRAU (Source: - American Chemical Society)

NLM (National Library of Medicine): ID: (101671502)

Index Copernicus Value 2018: 88.52

Volume 10, Issue 4: July-August : 2021, 07-14
ISSN (Online): 2279-0594

ISSN (Print): 2589-8752

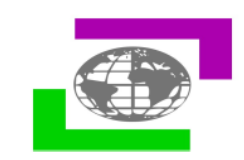

\title{
Drug Utilisation Pattern for Diabetic Foot
}

\section{Girish Gowda A*, M Kumarswamy, Rajesh Venkataramn, Mary Cheriyan, Asha Shaji}

SAC College of Pharmacy, B G Nagara-571448

\author{
Article Info: Received 20 June 2021; Accepted 29 July 2021 \\ DOI: https://doi.org/10.32553/jbpr.v10i4.872 \\ Corresponding author: Girish Gowda A \\ Conflict of interest statement: No conflict of interest
}

\begin{abstract}
Introduction: Diabetic mellitus is a chronic disorder which is rapidly raising, major public health problem. Having DM are more prone to multifarious complication like diabetic foot ulcer, which is highly susceptible to infection. Prevalence of bacterial flora in foot ulcer of this study population indicate current medical scenario of increased multidrug resistant diabetic foot infection, appropriate selection of antibiotic plays important role in DFU management increasing prevalence of antibiotic resistance is a major factor for morbidity or mortality in DFU.

The main objective of this study was to asses microbial sensitive resistance to antibiotic and also to know the drug utilisation of antibiotics in DFU.

Method: A prospective observational study was carried out in 123 inpatients, after taking written informed consent from those met the study criteria. The study was done from October 2017 to march 2018 in surgery department. Patient case sheet were reviewed and assessed on daily basis. Culture report form and all other relevant data were collected to assess the utilisation of antibiotic, it's sensitivity and resistant interaction, drug interactions and ADR were assessed by using Micromedex and other resources available in the department

Result: In this study population, about $87(70.3 \%)$ patients were male and $36(29.27 \%)$ female. Mainly patients of age range $41-50$ is $47(38.21 \%)$ followed by $51-60$ is $33(26.83 \%)$ then $61-70$ is 23(18.70\%).Hospital

Conclusion: Diabetic foot infections are real public health problem and early diagnosis along with appropriate treatments are essential. Different bacterial profiles and antibiotic sensitivity were found in different DFU. Clinician should try to stay updated in antibiotic sensitivity and resistant pattern of common pathogen in their area for better therapeutic outcome and to minimize medication errors.
\end{abstract}

Keywords: Diabetic foot ulcer, sensitivity, resistant, utilization of antibiotics, therapeutic outcome.

\section{Introduction}

Diabetes mellitus (DM) is one of the most prevalent pathology of endocrine that leads to a number of complications. According to the International Diabetes Federation (IDF) 2017, estimation of, epidemic proportion shows 425 million people in the world has diabetes and is gradually increase to 629 million in the year2045(younis2018). The major causes of DM involves impaired insulin secretion or inadequate sensitivity to secreted insulin. ${ }^{1} \mathrm{DM}$ is provoked mainly by metabolic complications, Such as due to improper storage 
and mobilization of metabolic fuels, including the catabolism (breakdown) and anabolism (buildup) of carbohydrates, lipids and proteins arise from defective insulin secretion, its action, or both that can gradually lead to immature death . ${ }^{2}$

The global prevalence of Diabetic foot ulcer (DFU) was $6.3 \%$ and it was more common in people with type 2 diabetes (younis2018). Frequently occurring complication normally affect lower extremities among which DFU is the recognised one. ${ }^{3}$ It remains prevalent and faces many challenges to treat and may results to hospital admissions and even amputations.It contributes the economic drain on the health care system which may tends to morbidity and even $\mathrm{s}$

The prevalence of causative organisms in DFI may vary from area to area. The organisms that occur on foot infections are mainly Staphylococcus aureus and Streptococcus pyrogens obtained from the patients' own skin and Enterococci from bowel. Among the Gram positive aerobes Staphylococci are more universal. Empirical antibiotic therapy should be optimized for local clinical microbiology,Many of these microorganisms are developing resistance to regularly used antibiotics largely due to their indiscriminate use. ${ }^{\mathbf{5 , 6}}$ The standard guidelines encircle with a combination of diabetes education, daily self foot checks, custom padded insoles, specialized diabetic shoes and routine physician follow-ups for foot inspections. ${ }^{7}$

In this present study we used to found out the most commonly found causative organism, prescription pattern of drugs used in diabetic foot along with its bacterial resistance and sensitivity.

\section{Methodology}

Materials Medical records of patients with diabetic foot ulcers, who were admitted at the Sri Adichunchanagiri Hospital And Research Center (AHRC) between October 2017 to March 2018, were reviewed. At the first visit a complete medical history was obtained. For analysis of the demographic and clinical characteristics, variables such as age, gender, smoking history, family history, HbA1C, wound duration, diabetes medications used, previous infection history and previous use of antibiotics were investigated, only the first admission period was included in this study. This study protocol was approved by the Institutional Review Board, AH\&RC, B G. Nagara.

(AHRC No: AIMS/IEC/1637/2017-18) Culture specimens were collected using sterile cotton swabs. Swabbing was done on inflammmed tissue as bacteria tend to present in greater number in these areas After rinsing the wound area with saline and debriding the wound, swab/ tissue samples were collected and taken to the microbiology laboratory.

The study data was analysed by using suitable descriptive statistics like mean, frequency, percentage etc, through Microsoft excels

\section{Result}

Table 1: Distribution lof Patients According to Age:

\begin{tabular}{|l|l|l|}
\hline Age & No of persons & \% Total \\
\hline $31-40$ & 9 & $\mathbf{7 . 3 2 \%}$ \\
\hline $41-50$ & 47 & $\mathbf{3 8 . 2 1 \%}$ \\
\hline $51-60$ & 33 & $\mathbf{2 6 . 8 3 \%}$ \\
\hline $61-70$ & 23 & $\mathbf{1 8 . 7 0 \%}$ \\
\hline$>70$ & 11 & $\mathbf{8 . 9 4 \%}$ \\
\hline
\end{tabular}


Among the 123 study population majority of the subjects were under the age group of 41-50 years i.e. in $47(38.21 \%)$ patients; followed by age group of 51-60 years in $33(26.83 \%)$ patients and age group of 61-70 years in
23(18.70\%) patients and 11 patients were under the age group of $>70$ years and 9 were under the age group of 31-40 years as shown in Figure 1.

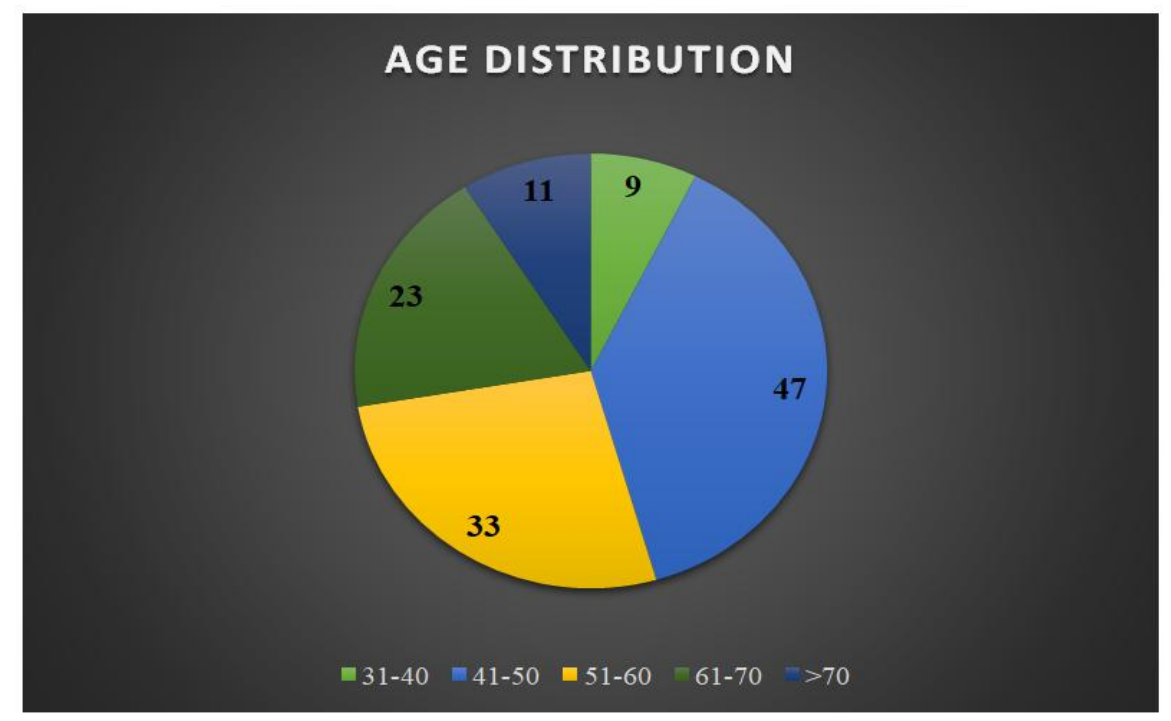

Figure1: Age Distribution

Table 2: Gender Distribution

\begin{tabular}{|l|l|l|}
\hline Gender & No of persons & \% Total \\
\hline Male & 87 & $\mathbf{7 0 . 7 3 \%}$ \\
\hline Female & 36 & $29.27 \%$ \\
\hline
\end{tabular}

Among 123 study population, 87(70.73\%) patients were males and 36(29.27\%) patients were females as shown in Figure 2.

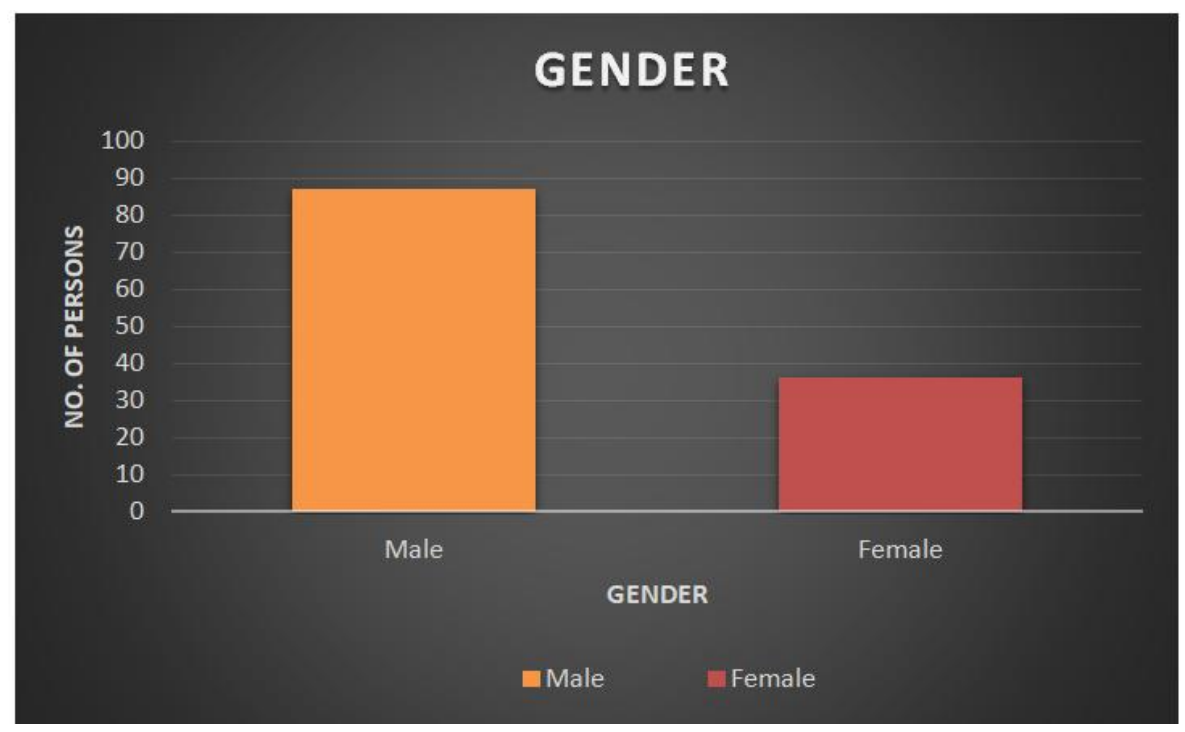

Figure 2: Gender Distribution 
Table 3: Culture Sensitivity Test

\begin{tabular}{|l|l|l|}
\hline Culture Sensitivity & No of persons & \% Total \\
\hline Done & 109 & $88.62 \%$ \\
\hline Not Done & 14 & $11.38 \%$ \\
\hline
\end{tabular}

Out of 123 patients, 109 (88.62\%) persons had done culture sensitivity test and 14(11.38\%) had not done the culture sensitivity test as shown in table 9.

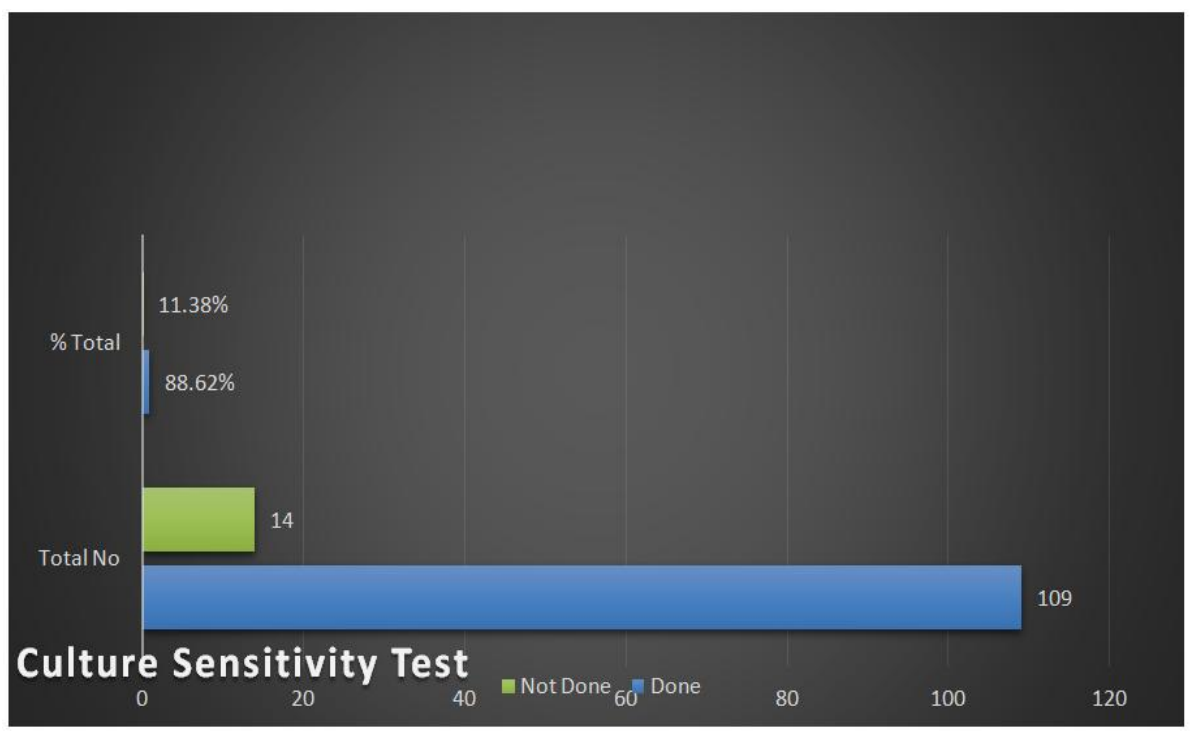

Figure 3: Culture Sensitivity Test

Table 4: Commonly Found Causative Organisms

\begin{tabular}{|l|l|l|}
\hline CAUSATIVE ORGANISM & TOTAL NO & \% TOTAL \\
\hline No organism & 44 & $35.77 \%$ \\
\hline Klebsiella species & 14 & $11.38 \%$ \\
\hline Citrobacter koseri & 2 & $1.63 \%$ \\
\hline Enterococcus species & 2 & $1.63 \%$ \\
\hline E coli & 6 & $4.88 \%$ \\
\hline MRS & 26 & $21.14 \%$ \\
\hline Non fermenting Gram -ve bacilli & 6 & $4.88 \%$ \\
\hline citrobacter species & 3 & $2.44 \%$ \\
\hline Providencia species & 5 & $4.07 \%$ \\
\hline Pseudomonas aeuroginosa & 7 & $5.69 \%$ \\
\hline Coagulase -ve staphylococcus & 3 & $2.44 \%$ \\
\hline Straptococcus aureus & 5 & $4.07 \%$ \\
\hline
\end{tabular}


Among 123 persons, 44 persons had been found without any causative organisms, 26 persons had found with MRS, 14 persons had been found with Klebsiella species, 7 persons had been found with Pseudomonas aeuroginosa, 6 persons had been found with $E$ coli and 6 persons had been found with Non fermenting,5 persons had been found with
Providencia species,5persons had been found with Streptococcus aureus,3persons had been found with Citrobacter species, 3 persons had been found with Coagulase -ve staphylococcus, 2 persons had been Citrobacter koseria and 2 persons had been found with Enterococcus species as shown in table 10.

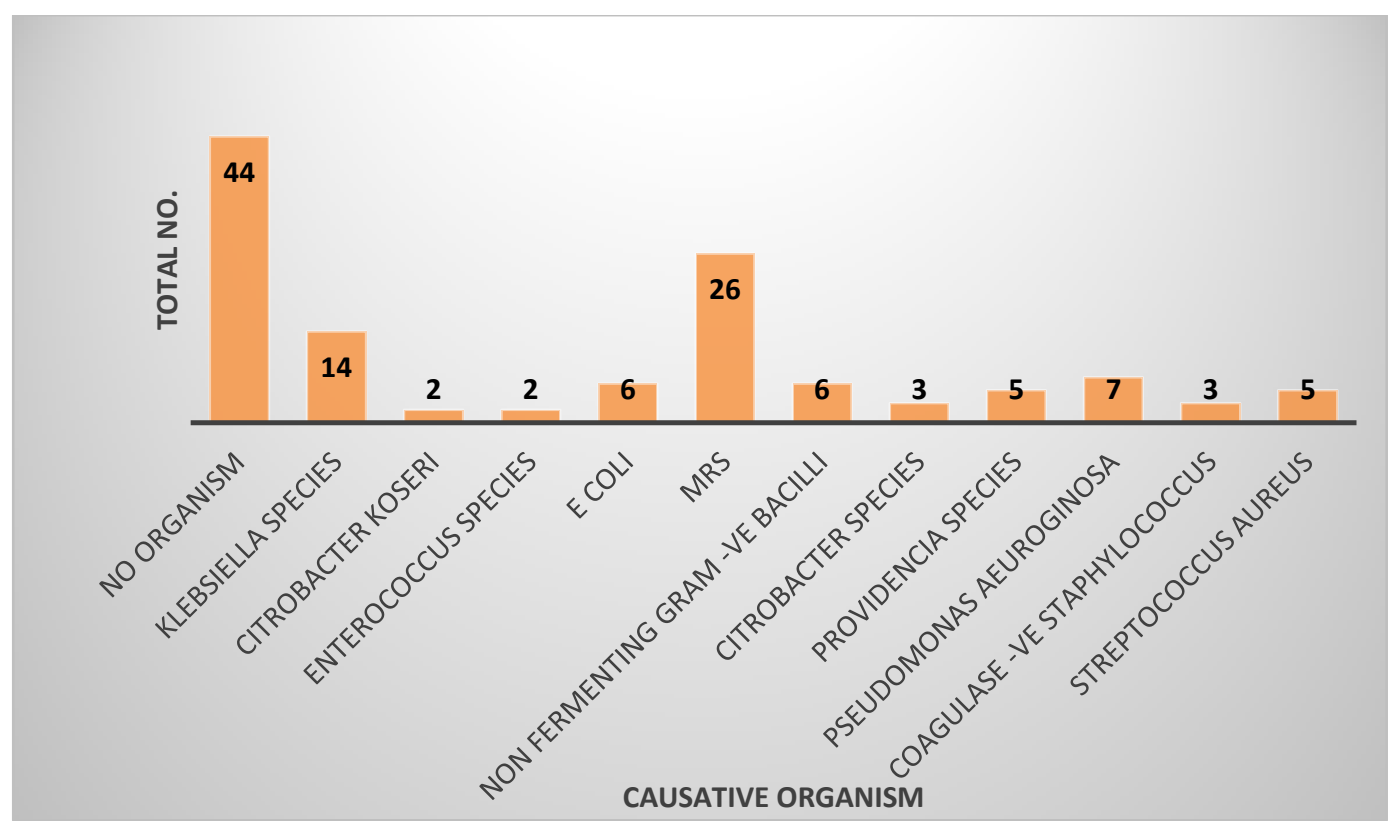

Figure 4: Commonly Found Causative Organisms

. Table 5: Commonly Used Antibiotics

\begin{tabular}{|lll|}
\hline Antibiotics & No of patients & \% Total \\
\hline Meropenem & $\mathbf{1 2}$ & $\mathbf{6 . 0 6 \%}$ \\
\hline Linezolid & $\mathbf{8}$ & $\mathbf{4 . 0 4 \%}$ \\
\hline Levofloxacin & $\mathbf{6}$ & $\mathbf{3 . 0 3 \%}$ \\
\hline Cefaperazone sodium+ Tazobactum & $\mathbf{6}$ & $\mathbf{3 . 0 3 \%}$ \\
\hline Cefaperazone sodium+ Sulbactum & $\mathbf{6}$ & $\mathbf{3 . 0 3 \%}$ \\
\hline Ceftriaxone & $\mathbf{5 2}$ & $\mathbf{2 6 . 2 6 \%}$ \\
\hline Metronidazole & $\mathbf{2 7}$ & $\mathbf{1 3 . 6 4 \%}$ \\
\hline Feropenem & $\mathbf{3}$ & $\mathbf{1 . 5 2 \%}$ \\
\hline Amikacin & $\mathbf{1 9}$ & $\mathbf{9 . 6 0 \%}$ \\
\hline Amoxicillin +Augmentin & 15 & $\mathbf{7 . 5 8 \%}$ \\
\hline Tinidizole & $\mathbf{2}$ & $\mathbf{1 . 0 1 \%}$ \\
\hline Piperacillin+Tazobactum & $\mathbf{4}$ & $\mathbf{2 . 0 2 \%}$ \\
\hline Cefaperazone & 11 & $\mathbf{5 . 5 6 \%}$ \\
\hline Cefixime & 17 & $\mathbf{8 . 5 9 \%}$ \\
\hline Glanocef X & 4 & $\mathbf{2 . 0 2 \%}$ \\
\hline Gentamycin & 6 & $\mathbf{3 . 0 3 \%}$ \\
\hline
\end{tabular}


Out of 123 prescriptions, 52 persons were taking Ceftriaxone, 27 persons were taking Metronidazole, 19 were taking Amikacin, 17 were taking Cefixime, 15 were taking Amoxicillin+ Clavulanic acid and 12 were taking Meropenem, 11 were taking Cefaperazone sodium, 8 were taking Linezolid,
6 were taking Cefaperazone sodium and tazobactum, 6 were taking Cefaperazone sodium and sulbactum, 6 were taking Gentamycin,6 were taking Levofloxacin,4 were taking Glanocef $\mathrm{X}, 4$ were taking Piperacillin and tazobactum, 3 were taking Feropenem and 2 were taking Tinidizole as shown in figure 3.

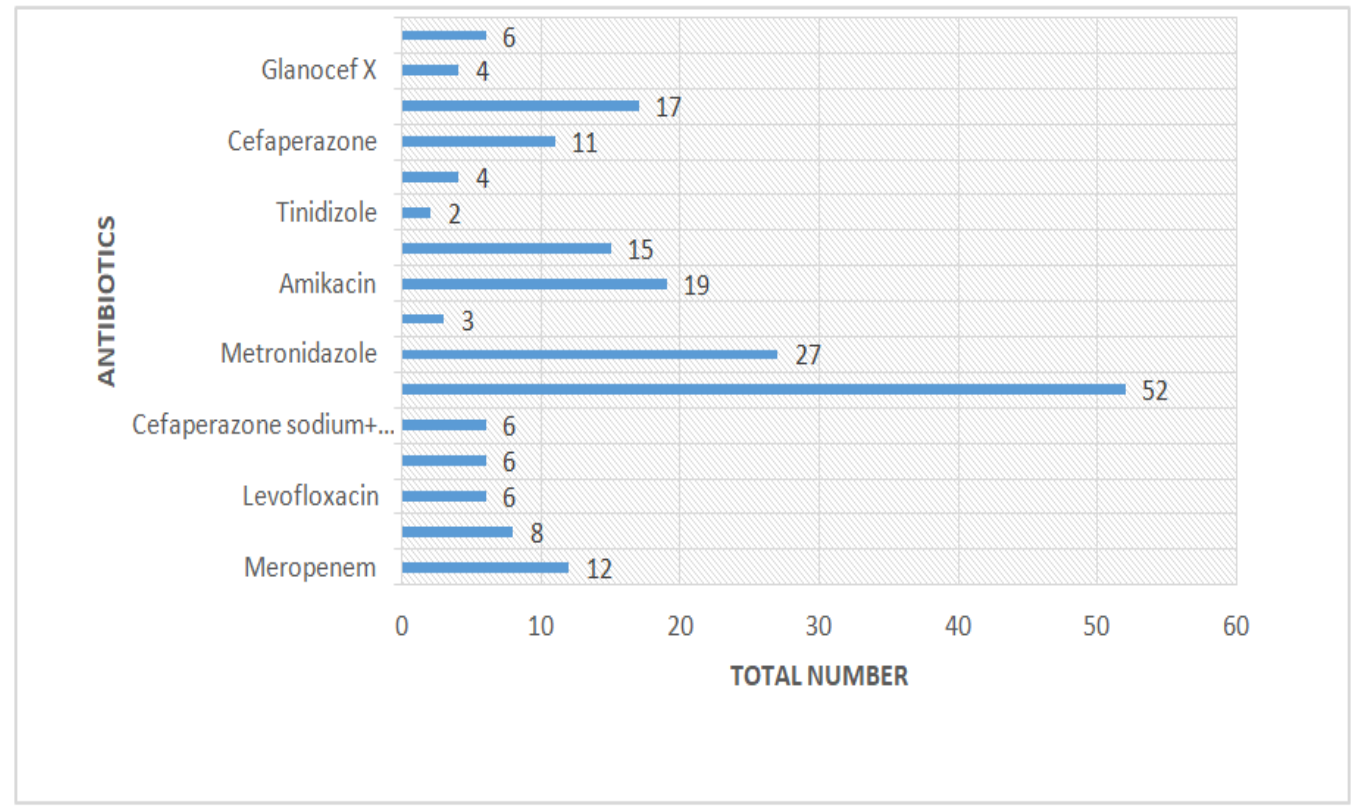

Figure 5: Commonly Used Antibiotics

Table 6: Drug Utilization Pattern

\begin{tabular}{|l|l|l|}
\hline Category & No.of drugs & Percentage \\
\hline Antibiotics & 198 & $49.13 \%$ \\
\hline Analgesics & 127 & $31.51 \%$ \\
\hline Vitamin supplements & 53 & $13.15 \%$ \\
\hline Others & 25 & $6.20 \%$ \\
\hline
\end{tabular}

\section{Discussion}

Among 123 patients enrolled in the study,age group of $41-50(31.21 \%)$ years patient were found to be more followed by 51-60(26.83\%) year,61-70(18.70\%) year,above $70(8.94 \%)$ year and were found in $31-40(7.32 \%)$ year. Male patient were higher affected $87(70.73 \%)$ while female $36(29.27 \%)$. The study was found to be similar to that conducted by Elhami E,Nagaraju K.,as DFU was found to be higher in age group of $40-50$ years, $59.33 \%$ males were affected where as $40.66 \%$ of female. Another study concluded by Venkatramana Manda etal.,also shares similar result in which $75 \%$ where male and the rest $25 \%$ femle.50-59 year $(42.5 \%)$ were more prone and least affected to age group of 20-29 years. One more study by Mithun N Oswal also gives matching result as mainly affected to age group of 41-50 years.

Of 123 subject enrolled in the study, majority of the patient's duration of hospital stay was found to more than 10 days $(97.56 \%)$ which have similar result with Perins.M.C etal.,it shows duration of more than 10 days 
Out of 123 patients involved in the study $109(86.62 \%)$ patients had done culture sensitivity test and 14(11.38\%) had not. And from culture test about 44 test given no organisms and among rest result, most commonly found organism was found to be MRSA(methicillin resistant staphylococcus aureus) which shows similar results with son $\mathbf{t} \mathbf{s}$ et al.,,study. This study shows most commonly found organisms as MRSA (methicillin resistant staphylococcus aureus(13.7\%). Study concluded by Hena.v etal.,, also found as the most frequent occurring causative organism as Staphylococcus.aureus which was as similar in study by Khare J etal.,

Among 123 patients in our study commonly prescribed drugs category were found to be antibiotics 198(79.13\%), analgesics $127(37.51 \%)$, vitamin supplements 53(13.155) and others $25(6.20 \%)$ which as similar result with study conducted by Peter $\mathbf{N}$, Nisssy Cherian., as antibiotics 211(34.9\%), analgesics 76(12.6\%), anti ulcerent 86(14.2\%), anti pyretic26(4.3\%), vitamine supplements $112(18.55)$ and others $92(15.2 \%)$.

In the present study the most commonly used antibiotics was found to be ceftriaxone $52(26.26 \%)$ followed by metronidazole 27 (13.64\%) and then amikacin 19 (9.60\%)which shows similar resulted by the study concluded by Elhami E,Nagaraju K.

Among all prescribed drugs,antibiotics were found to be 198(49.13\%) ,analgesics $127(31.51 \%)$,vitamin supplements $53(13.15 \%)$ and other drugs were about $25(6.20 \%)$ as shown in table no 5

\section{Conclusion}

This study had provided base line data regarding the prescription pattern (utilisation) of antibiotic drugs in diabetic foot patients. Total 65 culture were isolated. Among these, gram positive methicillin resistant staphylococcus $28(68.29 \%)$ and gram negative klebsiella $12(50 \%)$ spatients were prescribed with three drug combination due to their severity.

\section{Acknowledgement:}

The authors are grateful to the ministry of manpower for supporting the proposed research activity and to the higher management, faculty and doctors of surgery Department of AHRC, for their continuous support and encouragement and for providing the necessary infrastructure facilities for executing this work.

\section{References:}

1. Ibrahim A.J.K, Bhatawadekar S.M, Arunima, et.al., Bacterial profile of diabetic foot ulcer: study from western India. International journal of Health sciences and Research.2016; 6(5):65-71.

2. Garg R, Datta P, Gupta V, et.al., Anaerobic bacteriological profile of infected diabetic foot ulcers with their Antimicrobial susceptibility pattern: Need of the Hour. National Journal of Laboratory Medicine.2017; 6(3):1-4.

3. Edan A, Kareem A, Mary A., Bacterial wound Infections in diabetic patients and their therapeutic Implications. Medical practice and Review.2010; 1(2):12-15.

4. Ali O, Ali H.A, Southy H.E, et.al., Microbiological profile of Diabetic foot ulcer and use of IL6 as a predictor for Diabetic foot infection. International Journal of current Microbiology and Applied sciences.2016; 5(12):1-10.

5. Dwedar R, Ismail D.K, Abdulbaky A., Diabetic foot infection: Microbiological causes with special reference to their Antibiotic Resistance pattern. Egyptian Journal of Medical Microbiology.2015; 24(3):95-102.

6. Perim M.C, Borges J.D.C, Celeste S.R.C,et.al., Aerobic bacterial profile and Antibiotic Resistance in patients with diabetic foot infections. Revista da Sociedade Brasileria de Medicinal Tropical.2015; 48(5):546-54.

7. Tamalli M, Gamal M.A.B, Alghazal M.A., Common Aerobic bacterial Isolates from Diabetic foot ulcer and their Antibiotic susceptibility Testing. World 
Journal of Pharmacy and Pharmaceutical sciences.2015; 4(8):260-66.

8. Birra V, Hanumanthu A.K, Budati S, et.al., Aerobic bacterial profile and antibiotic resistance in patients with diabetic and non-diabetic wound infections. Journal of Evolution Med.Dent.Sci.2016; 5(98):7180-84.

9. Khare J, Srivatsava P, Khare J, et.al., Microbiological profile of Diabetic foot ulcers-Experience from a Teritiary care centre in South Asia. International Journal of General Medicine and Surgery.2017; 1(2):1-7.

10. Kunjappan S.P, Saju I.M., Aerobic bacterial profile of Diabetic ulcer in a Tertiary care centre. Journal of evolution Med.Dent.Sci.2017; 6(68):4859-62.

11. Sugandhi P, Prasanth D.A., Bacteriological profile of diabetic foot infections. International Journal of Innovative Research in science, engineering and technology.2014, 3(7): 14688-692.

12. Son S.T, Han S.K, Lee T.Y, et.al., The Microbiology of foot infections in Korea. Journal of wound management and Research.2017; 13(1):8-12.

13. Jithendranath A, Hegadi S, Bhai G, et.al., Bacteriological profile of Diabetic foot in a tertiary care centre in Trivandrum, India. Journal of current Microbiology and Applied Sciences.2016; 5(6):279-86.

14. Bengalorkar G.M, Kumar T.N., Culture and sensitivity pattern of micro-organism isolated from diabetic foot infections in a tertiary care hospital. International Journal of Biomedical and Pharmaceutical Research.2011; 1(2):3440.

15. JJ Mendes etal., clinical and bacteriological survey of diabetic foot infection in Lisbon; Diabetic research and clinical practice. 2012; 95(1): 153-161 